\title{
Vector-mean-field theory of the fractional quantum Hall effect
}

\author{
B. Rejaei and C. W. J. Beenakker \\ Instıtuut-Lorentz, Unvversity of Leiden, P.O. Box 9506, 2300 RA Leiden, The Netherlands
}

(Received 13 July 1992)

\begin{abstract}
A mean-field theory of the fractional quantum Hall effect is formulated based on the adiabatic principle of Greiter and Wilczek. The theory is tested on known bulk properties (excitation gap, fractional charge, and statistics), and then applied to a confined region in a two-dimensional electron gas (quantum dot). For a small number $N$ of electrons in the dot, the exact ground-state energy has cusps at the same angular momentum values as the mean-field theory. For large $N$, Wen's algebraic decay of the probability for resonant tunneling through the dot is reproduced, albeit with a different exponent.
\end{abstract}

Although the fractional quantum Hall effect (FQHE) in an unbounded, uniform two-dimensional (2D) electron gas is described accurately by Laughlin's variational wave functions, ${ }^{1}$ this theory is not easily applied to confined or nonuniform systems. The fact that it is now possible experimentally to study the FQHE in a nanostructured 2D electron gas calls for a mean-field theory which can explain the novel effects occuring in such "mesoscopic" systems. In a conventional mean-field treatment of the Coulomb interactions, however (such as the Hartree-Fock apploximation), the subtle correlations responsible for the incompressibility of the FQHE liquid are lost. In a recent paper, Greiter and Wilczek ${ }^{2}$ have proposed an "adiabatic principle" for the FQHE, which suggests a simple mean-field approximation that might be able to describe the FQHE in confined geometries. The adiabatic principle of Ref. 2 (summarized below) is based on the introduction of a fictitious long-range vector potential interaction between the electrons. By treating this interaction in mean-field theory one has a "vectormean-field theory" of the FQHE, a name borrowed ${ }^{3}$ from anyon superconductivity, ${ }^{4,5}$ where the fractional statistics is mediated by a similar gauge interaction.

In this paper we will show that the vector-mean-field theory reproduces the known bulk properties of the correlated FQHE states, such as fractional charge and statistics of the quasiparticle excitations, and we will calculate the excitation energies. These bulk properties are also well described by the Chern-Simon field theories of Refs. $6-10$, although, as far as we are aware, this is the first time that a mean-field theory is used to actually calculate the excitation gap. We will then focus on a simple confined geometry, a quantum dot with parabolic confinement. For a few electrons in the dot we compare the mean-field theory with the exact diagonalization of the Hamiltonian. Finally, we will consider the problem of tunneling through a quantum dot in the FQHE regime, in connection with the "orthogonality catastrophe" predicted recently by Wen ${ }^{11}$ and Kinaret et al. ${ }^{12}$

The adiabatic principle of Greiter and Wilczek ${ }^{2}$ is formulated in terms of the Hamiltonian

$$
\begin{aligned}
\mathcal{H}_{\lambda}= & \sum_{\imath} \frac{1}{2 m}\left[\mathbf{p}_{\imath}+e \mathbf{A}\left(\mathbf{r}_{\imath}\right)-e \lambda \sum_{\jmath \neq \imath)} \mathbf{a}\left(\mathbf{r}_{\imath}-\mathbf{r}_{\jmath}\right)\right]^{2} \\
& +\sum_{\imath<\jmath} u\left(\mathbf{r}_{\imath}-\mathbf{r}_{\jmath}\right)+\sum_{\imath} V\left(\mathbf{r}_{\imath}\right)
\end{aligned}
$$

where $\nabla \times \mathbf{A}=B \hat{\mathbf{z}}$ is the external magnetic field (with $\hat{z}$ the unit vector perpendicular to the $2 \mathrm{D}$ eléctron gas), and $V$ is the electrostatic potential from impurities or an external confinement. In addition to the ordinary interaction $u(\mathrm{r})$, the electrons interact via the vector potential $-\lambda \mathbf{a}(\mathbf{r})$, where

$$
\mathbf{a}(\mathbf{r})=\frac{h}{e} \frac{\hat{\mathbf{z}} \times \mathbf{r}}{2 \pi r^{2}}, \quad \nabla \times \mathbf{a}(\mathbf{r})=\frac{h}{e} \delta(\mathbf{r}) \hat{\mathbf{z}}
$$

The Hamiltonian $\mathcal{H}_{\lambda}$ is thus obtained from the ordinary Hamiltonian $\mathcal{H}_{0}$ by binding a flux tube of strength $-\lambda h / e$ to each electron. Greiter and Wilczek now propose the following adiabatic mapping: ${ }^{13}$ Starting with an eigenstate $\Psi_{0}$ of $\mathcal{H}_{0}$, which satisfies $\mathcal{H}_{0} \Psi_{0}=E_{0} \Psi_{0}$, one switches on the vector potential interaction adiabatically by increasing $\lambda$ from 0 to an even, positive integer $2 k$. After $\Psi_{0}$ has evolved adiabatically into $\Psi_{2 k}$, with $\mathcal{H}_{2 k} \Psi_{2 k}=E_{2 k} \Psi_{2 k}$, one eliminates the vector potential interaction in $\mathcal{H}_{2 k}$ by the gauge transformation $\chi_{2 k} \mathcal{H}_{2 k} \chi_{2 k}^{-1} \chi_{2 k} \Psi_{2 k}=\mathcal{H}_{0} \chi_{2 k} \Psi_{2 k}=E_{2 k} \chi_{2 k} \Psi_{2 k}$, where

$$
\chi_{2 k}=\prod_{\imath<\jmath} \frac{\left(z_{\imath}-z_{j}\right)^{2 k}}{\left|z_{\imath}-z_{\jmath}\right|^{2 k}}, \quad z_{\imath}=x_{\imath}-\imath y_{\imath} .
$$

Hence, $\Psi_{0}$ is mapped onto $\chi_{2 k} \Psi_{2 k}$, a new, exact eigenstate of the original Hamiltonian $\mathcal{H}_{0}$. Motivated by Jain's theory of the FQHE, ${ }^{14}$ Greiter and Wilczek propose that the incompressible FQHE states can be obtained by an adiabatic mapping of the incompressible states of the integer QHE (IQHE).

The mean-field approximation to the adiabatic mapping described above is suggested by the vector-meanfield theory of anyon superconductivity. ${ }^{3-5}$ In this approximation the flux tubes are smeared out, yielding a fictitious magnetic field $\mathbf{B}^{f}$ proportional to the electron 
density. In addition, a fictitious electric field $\mathbf{E}^{f}$ is generated by the motion of the flux tubes bound to the electrons. ${ }^{15}$ The mean-field Hamiltonian is

$\mathcal{H}_{\lambda}^{\mathrm{MF}}=\frac{1}{2 m}\left(\mathbf{p}+e \mathbf{A}-e \lambda \mathbf{A}^{f}\right)^{2}+e \lambda \Phi^{f}+U+V$.

The fictitious potentials $\mathbf{A}^{f}$ and $\Phi^{f}$ are given by

$$
\begin{aligned}
& \mathbf{A}^{f}(\mathbf{r})=\int d \mathbf{r}^{\prime} \mathbf{a}\left(\mathbf{r}-\mathbf{r}^{\prime}\right) n\left(\mathbf{r}^{\prime}\right), \\
& \Phi^{f}(\mathbf{r})=\int d \mathbf{r}^{\prime} \mathbf{a}\left(\mathbf{r}-\mathbf{r}^{\prime}\right) \cdot \mathbf{j}\left(\mathbf{r}^{\prime}\right),
\end{aligned}
$$

and the ordinary Hartree potential is given by

$$
U(\mathbf{r})=\int d \mathbf{r}^{\prime} u\left(\mathbf{r}-\mathbf{r}^{\prime}\right) n\left(\mathbf{r}^{\prime}\right) .
$$

The electron density $n$ and current density $\mathbf{j}$ are to be determined self-consistently from the eigenfunctions $\psi_{\lambda, i}(i=1, \ldots, N)$ of $\mathcal{H}_{\lambda}^{\mathrm{MF}}$. After increasing $\lambda$ from 0 to $2 k$, the mean-field state is given by $\Psi^{\mathrm{MF}}=\chi_{2 k} \Psi_{2 k}^{\mathrm{MF}}$, where $\Psi_{\lambda}^{\mathrm{MF}}$ is the Slater determinant of $\psi_{\lambda, i}$. A further simplification results if the potential $U$ is also switched on adiabatically by the substitution $U \rightarrow(\lambda / 2 k) U$. Then, $\mathcal{H}_{0}^{\mathrm{MF}}$ describes a system of noninteracting electrons, so that the initial state $\Psi_{0}^{\mathrm{MF}}$ of the mapping can be determined exactly.

The mean-field equations can be solved analytically if the initial state $\Psi_{0}^{\mathrm{MF}}$ consists of $p$ completely filled Landau levels. We can either consider the case $u(\mathbf{r})$ short ranged with $V(\mathbf{r}) \equiv 0$, or the Coulomb potential $u(\mathbf{r})=$ $e^{2} / r$ with $V(\mathbf{r})$ the potential of a neutralizing background of positive charges with density $\rho=n$. In both cases the electron density remains uniform as we switch on the interactions. The fictitious magnetic field is also uniform, since $B^{f} \hat{\mathbf{z}}=\nabla \times \mathbf{A}^{f}=(h / e) n \hat{\mathbf{z}}$. Since the mapping is adiabatic, no transitions occur between different Landau levels. Therefore, we retain $p$ fully filled Landau levels, but now in the effective magnetic field $B^{\text {eff }}=B-\lambda B^{f}=$ $B-\lambda(h / e) n$. Equating $B^{\text {eff }}=h n / e p$, we find $n=p(\lambda p+$ $1)^{-1}(e B / h)$. The eigenfunctions of $\mathcal{H}_{\lambda}^{\mathrm{MF}}$ (for quantum numbers $n, m=0,1,2, \ldots)$ are

$\psi_{\lambda, n, m}=\frac{(-\mathrm{i})^{n}(n !)^{1 / 2}}{(2 \pi m !)^{1 / 2} \ell_{\lambda}} \zeta^{m-n} L_{n}^{m-n}\left(|\zeta|^{2}\right) \exp \left(-\frac{|\zeta|^{2}}{2}\right)$

where $\ell_{\lambda}=(\lambda p+1)^{1 / 2}(\hbar / e B)^{1 / 2}$ is the effective magnetic length, $L_{n}^{m-n}$ is the Laguerre polynomial, and $\zeta \equiv 2^{-1 / 2} z / \ell_{\lambda}$. After letting $\lambda \rightarrow 2 k$ we recover
Jain's formula ${ }^{14}$ for the hierarchy of FQHE filling factors $\nu=p(2 k p+1)^{-1}$.

For a Coulomb potential with a neutralizing background, the interaction energy of the system after subtracting the background contributions is

$$
\begin{aligned}
& E_{\mathrm{int}}^{\mathrm{MF}}=-\frac{e^{2}}{2} \int d \mathbf{r} \int d \mathbf{r}^{\prime} \frac{\left|D\left(\mathbf{r}, \mathbf{r}^{\prime}\right)\right|^{2}}{\left|\mathbf{r}-\mathbf{r}^{\prime}\right|} \\
& D\left(\mathbf{r}, \mathbf{r}^{\prime}\right)=\sum_{i=1}^{N} \psi_{2 k, i}(\mathbf{r}) \psi_{2 k, i}^{*}\left(\mathbf{r}^{\prime}\right) .
\end{aligned}
$$

From Eqs. (8) and (9) we find, for $\lambda=2 k$,

$$
\begin{aligned}
\frac{\ell_{0}}{e^{2}} \frac{E_{\text {int }}^{\mathrm{MF}}}{N}=\frac{-(\pi / 8)^{1 / 2}}{p(2 k p+1)^{1 / 2}} \sum_{i, j=0}^{p-1}\left(\begin{array}{c}
p \\
i+1
\end{array}\right)\left(\begin{array}{c}
p \\
j+1
\end{array}\right) \\
\times \frac{(-1)^{i+j}(2 i+2 j) !}{4^{i+j} i ! j !(i+j) !},
\end{aligned}
$$

where $\ell_{0} \equiv(\hbar / e B)^{1 / 2}$. In Table I we have listed the interaction energy per electron for $k=1, p=1,2$, together with the exact results ${ }^{16}$ for $\nu=\frac{1}{3}$ and $\frac{2}{5}$. The mean-field values are too large by about $10 \%$.

To determine whether the mean-field ground state $\Psi^{\mathrm{MF}}$ can be characterized as a mean-field FQHE state, we have to study its excitations. The charged FQHE excitations should have a gap, and fractional charge and statistics. ${ }^{1}$ We assume that the adiabatic mapping carries the particle and hole excitations of the IQHE into the quasiparticle and quasihole excitations of the FQHE. The elementary charged excitations of the IQHE at filling factor $p$ have an electron in the $(p+1)$ th Landau level or a hole in the $p$ th Landau level. Here, unlike the previous case, the self-consistent mean-field equations do not allow an analytic solution and we had to solve them numerically. Our numerical method will be discussed in detail elsewhere. Here, we only give the results of the calculation carried out for $N \simeq 40$. The (gross) quasiparticle $\left(\epsilon_{-}\right)$and quasihole $\left(\epsilon_{+}\right)$energies, as well as the excitation gap energy $\epsilon_{\mathrm{g}}=\epsilon_{-}+\epsilon_{+}$, are compared with exact results ${ }^{16}$ in Table I. There is reasonable agreement for the quasiparticle and quasihole energies of the $\frac{1}{3}$ state and the quasihole energy of the $\frac{2}{5}$ state. The result for the $\frac{2}{5}$ quasiparticle is less satisfactory.

The quasiparticle (quasihole) charges can be calculated from the mean-field density profiles. For example, for the $\frac{1}{3}$ state we find $Q_{-}=-0.374 e$ and $Q_{+}=0.295 e$. The deviation from the exact fraction $\pm e / 3$ is due to the finite

TABLE I. Comparison of the mean-field (MF) results with the exact calculations (ex) (Ref. 16). The interaction energy per particle $\left(E_{\mathrm{int}} / N\right)$, and the (gross) quasiparticle $\left(\epsilon_{-}\right)$, quasihole $\left(\epsilon_{+}\right)$, and excitation-gap $\left(\epsilon_{g}\right)$ energies are compared for $\nu=\frac{1}{3}(k=1, p=1)$ and $\nu=\frac{2}{5}(k=1, p=2)$. The energy unit is $e^{2} / \ell_{0}$.

\begin{tabular}{lcccccccc}
\hline & \multicolumn{2}{c}{$E_{\text {int }} / N$} & \multicolumn{2}{c}{$\epsilon_{-}$} & \multicolumn{2}{c}{$\epsilon_{+}$} & \multicolumn{2}{c}{$\epsilon_{g}$} \\
$\nu$ & $\mathrm{MF}$ & $\mathrm{ex}$ & $\mathrm{MF}$ & $\mathrm{ex}$ & $\mathrm{MF}$ & $\mathrm{ex}$ & $\mathrm{MF}$ & $\mathrm{ex}$ \\
\hline$\frac{1}{3}$ & -0.362 & -0.410 & -0.104 & -0.130 & 0.211 & 0.232 & 0.107 & 0.102 \\
$\frac{2}{5}$ & -0.385 & -0.434 & -0.063 & -0.084 & 0.150 & 0.145 & 0.087 & 0.061 \\
\hline \hline
\end{tabular}


number of electrons in the calculation. We now argue that in the limit $N \rightarrow \infty$, the mean-field values become identical to the exact fractions. Consider an IQHE excitation of charge $\pm e$ at the origin. As one switches on the interactions, the electrons far from the origin see an excess flux $\phi_{ \pm}=\lambda Q_{ \pm}\left(h / e^{2}\right)$ at the origin, because of the presence of an excitation of charge $Q_{ \pm}$. The excess flux shifts the single-electron wave functions outwards or inwards (depending on the sign), in such a way that an excess charge $\delta Q_{ \pm}=-p \phi_{ \pm} /\left(h / e^{2}\right)$ is induced near the origin. Thus, the net charge of the excitation becomes $Q_{ \pm}= \pm e+\delta Q_{ \pm}$, which implies $Q_{ \pm}= \pm e(2 k p+1)^{-1}$ (after $\lambda \rightarrow 2 k$ ). For $k=1, p=1,2$ we recover the wellknown results $Q_{ \pm}= \pm e / 3, Q_{ \pm}= \pm e / 5$ for the $\frac{1}{3}$ and $\frac{2}{5}$ states, respectively. ${ }^{17}$ The fractional statistics follows directly from the fractional charge, ${ }^{18}$ the statistical phase acquired upon exchanging two quasiparticles (quasiholes) being $\pm \pi(2 k p+1)^{-1}$.

Now that we have shown that the vector-mean-field theory describes the FQHE in unbounded homogeneous systems, we turn to confined inhomogeneous systems. We have solved the mean-field equations for a quantum dot with a parabolic confining potential $V(r)=\frac{1}{2} m \omega_{0}^{2} r^{2}$, starting from an initial incompressible IQHE state. We have also calculated the exact ground-state energies for $N=5$ and 6 , by diagonalizing the Hamiltonian $\mathcal{H}_{0}$ in the lowest Landau level (in the translational-invariant subspace, following the method of Ref. 19). In Fig. 1 we have plotted the mean-field and exact electronelectron interaction energies $E_{e e}$ as functions of the total angular momentum $L$ of the system. The total interaction energy (including the confinement energy) is given by $E_{\text {int }}=E_{e e}+\frac{1}{2} \hbar(L+N)\left[\left(\omega_{\mathrm{c}}^{2}+4 \omega_{0}^{2}\right)^{1 / 2}-\omega_{\mathrm{c}}\right]$, where $\omega_{\mathrm{c}}=e B / \mathrm{m}$. The exact diagonalization yields a value of $E_{e e}$ for each integer $L$ (open symbols in Fig. 1). Squares represent the incompressible states, i.e., which are a ground state for some strength $\omega_{0}$ of the external confinement. ${ }^{19}$ The states which are not stable under external confinement are represented by circles. The adiabatic mapping, in contrast, yields only particular values of $L$ (triangles). One sees from Fig. 1 that the exact ground-state energy shows a strong cusp at these values, and the system becomes incompressible except at $N=5, L=22$. We conclude that the angular momentum values reached by the adiabatic mapping correspond to cusps in the interaction energy, and therefore are good candidates for incompressibility.

As a final application of the vector-mean-field theory, we consider the orthogonality catastrophe for resonant tunneling through a quantum dot in the FQHE regime. It has been shown by Wen ${ }^{11}$ and by Kinaret et al. ${ }^{12}$ that the resonant conductance peaks of a quantum dot in the $\frac{1}{m}$ FQHE state (in the regime of thermally broadened resonances) are suppressed in the limit $N \rightarrow \infty$. The tunneling probability ${ }^{20}$ is proportional to $|\mathcal{M}|^{2}=\left|\left\langle\Psi_{N+1}\left|c_{\Delta L}^{\dagger}\right| \Psi_{N}\right\rangle\right|^{2}$, where $\Psi_{N}$ is the $N$-electron ground state (with angular momentum $L_{N}$ ), and the operator $c_{\Delta L}^{\dagger}$ creates an electron in the lowest Landau level with wave function $\psi_{\Delta L}$ and angular momentum $\Delta L=L_{N+1}-L_{N}$. In the IQHE, the overlap $\mathcal{M}$ is unity.

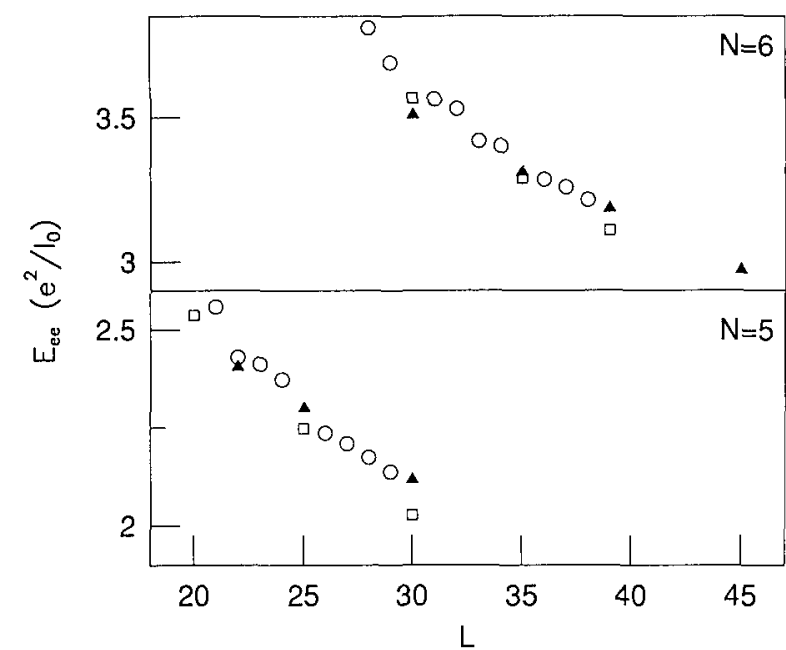

FIG. 1. Electron-electron interaction energy of five and six electrons as a function of the angular momentum $L$. Triangles follow from the adiabatic mapping in mean-field approximation. Squares and circles are exact results, squares representing incompressible ground states. Exact results for $N=6, L>39$ could not be obtained because of computational restrictions. The range $L \leq 21(N=5)$ and $L \leq 29(N=6)$ cannot be reached by adiabatic mapping.

Wen and Kinaret et al. find that, in the $\frac{1}{m}$ FQHE state, $|\mathcal{M}|^{2}$ vanishes algebraically as $N^{-(m-1) / 2}$ when $N \rightarrow \infty$, as a manifestation of the non-Fermi liquid nature of the FQHE.

To see whether the mean-field theory can reproduce this orthogonality catastrophe, we need the matrix element $\mathcal{M}^{\mathrm{MF}}=\left\langle\Psi_{N+1}^{\mathrm{MF}}\left|c_{\Delta L}^{\dagger}\right| \Psi_{N}^{\mathrm{MF}}\right\rangle$, which we rewrite as

$\mathcal{M}^{\mathrm{MF}}=\left\langle 0\left|\prod_{\imath=1}^{N+1} c_{\imath} \chi_{2 k, N+1}^{\dagger} c_{\Delta L}^{\dagger} \chi_{2 k, N} \prod_{\jmath=1}^{N} c_{\jmath}^{\dagger}\right| 0\right\rangle$.

Here, $c_{\jmath}^{\dagger}$ creates an electron in the eigenstate $\psi_{2 k, \jmath}$ of $\mathcal{H}_{2 k}^{\mathrm{MF}}$, and the operator $\chi_{2 k, N}$ carries out the gauge transformation (3) on an $N$-electron wave function. After substituting $c_{\Delta L}^{\dagger}=\int d \mathbf{s} \psi_{\Delta L}(\mathbf{s}) \psi^{\dagger}$ (s) (where $\psi^{\dagger}$ is the field operator in second quantization), we eliminate $\chi_{2 k, N}$ and $\chi_{2 k, N+1}^{\dagger}$, by using $\chi_{2 k, N+1}^{\dagger} \chi_{2 k, N+1}=1$, and the identity

$$
\psi^{\dagger}(\mathbf{s}) \chi_{2 k, N} \prod_{\jmath=1}^{N} c_{\jmath}^{\dagger}=\chi_{2 k, N+1} \psi^{\dagger}(\mathbf{s}) \prod_{\jmath=1}^{N} c_{\jmath}^{\dagger}(\mathbf{s}) .
$$

The operator $c_{\jmath}^{\dagger}(\mathbf{s})$ creates an electron with the wave function $S(\mathbf{r} ; \mathbf{s}) \psi_{2 k, j}(\mathbf{r})$, where

$S(\mathbf{r} ; \mathbf{s}) \equiv \frac{(z-\xi)^{-2 k}}{|z-\xi|^{-2 k}}, \quad z=x-i y, \xi=\mathbf{s}_{x}-i \mathbf{s}_{y}$.

Substitution into Eq. (10) leads to

$$
\begin{aligned}
& \mathcal{M}^{\mathrm{MF}}=\int d \mathbf{s} \psi_{\Delta L}(\mathbf{s}) \mathcal{M}(\mathbf{s}), \\
& \mathcal{M}(\mathbf{s})=\left\langle 0\left|\prod_{\imath=1}^{N+1} c_{\imath} \psi^{\dagger}(\mathbf{s}) \prod_{\jmath=1}^{N} c_{\jmath}^{\dagger}(\mathbf{s})\right| 0\right\rangle=\operatorname{det} \mathcal{M}_{\imath \jmath}(\mathbf{s}),
\end{aligned}
$$


where $\mathcal{M}_{\imath \jmath}(\mathbf{s})$ is a $(N+1) \times(N+1)$ matrix with elements

$$
\mathcal{M}_{\imath \jmath}(\mathbf{s})=\int d \mathbf{r} \psi_{2 k, \imath}^{*}(\mathbf{r}) S(\mathbf{r} ; \mathbf{s}) \psi_{2 k, \jmath}(\mathbf{r}) \quad(j \neq N+1)
$$

$$
\mathcal{M}_{2, N+1}(\mathbf{s})=\psi_{2 k, 2}^{*}(\mathbf{s}) .
$$

Since we are dealing with a finite system, the wave functions $\psi_{2 k, \imath}$ will differ from $\psi_{2 k, n, m}$ in Eq. (8). However, when $N$ is large, $\psi_{2 k, n, m}$ will be a good approximation. We can then calculate the determinant analytically. For the $\frac{1}{3}$ FQHE state the determinant is given by

$$
\begin{aligned}
& \mathcal{M}(\mathbf{s})=\frac{\left(\eta^{*}\right)^{3 N}|\eta|^{-2 N}}{(2 \pi N !)^{1 / 2} \ell_{2 k}} \exp \left(-\frac{|\eta|^{2}}{2}\right) f_{N}\left(|\eta|^{2}\right), \\
& f_{N}(x)=\int_{0}^{x} d t\left(1-\frac{t}{x}\right)^{2} \frac{t^{N-1} \exp (-t)}{(N-1) !}
\end{aligned}
$$

where $\eta=2^{-1 / 2} \xi / \ell_{2 k}$. After substituting into Eq. (13) and carrying out the integration we find $\left|\mathcal{M}^{\mathrm{MF}}\right|^{2} \simeq$ $0.380 N^{-2}$ for $N \gg 1$. We conclude that the mean-field theory reproduces the algebraic decay of the tunneling matrix element for large $N$, but with a different value of the exponent $\left(|\mathcal{M}|^{2} \propto N^{-2}\right.$ instead of $\propto N^{-1}$ in Refs. 11 and 12). In the present context, the orthogonality catastrophe originates from the correlations created by the gauge transformation $\chi$, required to remove the fictitious vector potential from the Hamiltonian (1).

In summary, we have investigated the adiabatic mapping of Greiter and Wilczek, ${ }^{2}$ by means of a mean-field approximation of the vector potential interaction. In con- trast to previous theories, this mean-field theory can easily be applied to confined geometries, such as a quantum dot. Starting from an unbounded incompressible state of noninteracting electrons, we have shown that the adiabatic mapping leads to a correlated state with the characteristics of the FQHE (excitation gap, fractional quasiparticle charge, and statistics). The non-Fermi-liquid nature of the mean-field ground state is illustrated by the algebraic suppression of the probability for resonant tunneling through the dot in the limit $N \rightarrow \infty$ (the orthogonality catastrophe of Wen ${ }^{11}$ and Kinaret et al. $\left.{ }^{12}\right)$.

We conclude by identifying some directions for future research. The shortcomings of the mean-field approach originate from the fact that it does not give the correct behavior of the wave function at short separations. This is particularly serious for properties involving the kinetic energy (such as cyclotron resonance). A projection onto the lowest Landau level (as in Jain's approach ${ }^{14}$ ) might improve the results, but would also make the theory less tractable. At present we can only reach filling factors $\nu<$ $\frac{1}{2}$ by adiabatic mapping. To study the interesting effects occuring at higher filling factors (in particular $\nu=\frac{2}{3}$, see Refs. 12 and 21) one would presumably have to invoke some form of particle-hole symmetry. The theory in its present form may well serve as a starting point for a study of interfacial effects, for example, the correlation energy and density profile at the interface between the $\nu=\frac{1}{3}$ and $\nu=\frac{2}{5}$ FQHE states.

This work has been supported in part by the Dutch Science Foundation NWO/FOM.
${ }^{1}$ R. B. Laughlin, Phys. Rev. Lett. 50, 1395 (1983).

${ }^{2}$ M. Greiter and F. Wilczek, Mod. Phys. Lett. B 4, 1063 (1990); Nucl. Phys. B 370, 577 (1992).

${ }^{3}$ C. Gros, S. M. Girvin, G. S. Canright, and M. D. Johnson, Phys. Rev. B 43, 5883 (1991).

${ }^{4}$ R. B. Laughlin, Phys. Rev. Lett. 60, 2677 (1988).

${ }^{5}$ A. L. Fetter and C. B. Hanna, Phys. Rev. B 45, 2335 (1992).

${ }^{6}$ S. C. Zhang, T. H. Hansson, and S. Kivelson, Phys. Rev. Lett. 62, 82 (1989).

${ }^{7}$ N. Read, Phys. Rev. Lett. 62, 86 (1989).

${ }^{8}$ A. Lopez and E. Fradkin, Phys. Rev. B 44, 5246 (1991).

${ }^{9}$ S. G. Ovchinnikov, Pis'ma Zh. Eksp. Teor. Fiz. 54, 579 (1991) [JETP Lett. 54, 583 (1991)].

${ }^{10}$ A. Perez Martinez and A. Cabo, Mod. Phys. Lett. B 5, 1703 (1991).

${ }^{11}$ X. G. Wen, Phys. Rev. B 41, 12838 (1990).

${ }^{12}$ J. M. Kinaret, Y. Meir, N. S. Wingreen, P. A. Lee, and X. G. Wen, Phys. Rev. B 45, 9489 (1992).

${ }^{13}$ There is a minor difference between the adiabatic mapping described here (which occurs at constant external magnetic field), and the mapping of Ref. 2. In addition to binding negative flux tubes to the electrons, Greiter and Wilczek increase the external magnetic field in such a way that the total flux through the system remains unchanged. In a uniform system, their mapping occurs at a constant electron density. In a nonuniform system, it would imply a nonuniform external magnetic field, which is why we have preferred the alternative formulation presented here.

${ }^{14}$ J. K. Jain, Phys. Rev. Lett. 63, 199 (1989).

${ }^{15}$ B. Rejaei and C. W. J. Beenakker, Phys. Rev. B 43, 11392 (1991).

${ }^{16}$ N. d'Ambrumenil and R. Morf, Phys. Rev. B 40,6108 (1989).

${ }^{17}$ The Quantum Hall Effect, edited by R. E. Prange and S. M. Girvin (Springer, New York, 1987).

${ }^{18}$ D. Arovas, J. R. Schrieffer, and F. Wilczek, Phys. Rev. Lett. 53, 722 (1984).

${ }^{19}$ S. A. Trugman and S. Kivelson, Phys. Rev. B. 31, 5280 (1985).

${ }^{20}$ Y. Meir and N. S. Wingreen, Phys. Rev. Lett. 68, 2512 (1992).

${ }^{21}$ M. D. Johnson and A. H. MacDonald, Phys. Rev. Lett. 67, 2060 (1991). 